ARTICLE

Received 16 Jan 2015 | Accepted 24 Jun 2015 | Published 29 Jul 2015

DOI: $10.1038 /$ ncomms 8905

OPEN

\title{
Band structure engineering via piezoelectric fields in strained anisotropic CdSe/CdS nanocrystals
}

\author{
Sotirios Christodoulou1,2, Fernando Rajadell ${ }^{3}$, Alberto Casu', Gianfranco Vaccaro ${ }^{4}$, Joel Q. Grim', \\ Alessandro Genovese ${ }^{1}$, Liberato Manna ${ }^{1}$, Juan I. Climente ${ }^{3}$, Francesco Meinardi ${ }^{4}$, Gabriele Rainò ${ }^{5}$, \\ Thilo Stöferle ${ }^{5}$, Rainer F. Mahrt ${ }^{5}$, Josep Planelles ${ }^{3}$, Sergio Brovelli ${ }^{4} \&$ Iwan Moreels ${ }^{1}$
}

\begin{abstract}
Strain in colloidal heteronanocrystals with non-centrosymmetric lattices presents a unique opportunity for controlling optoelectronic properties and adds a new degree of freedom to existing wavefunction engineering and doping paradigms. We synthesized wurtzite CdSe nanorods embedded in a thick CdS shell, hereby exploiting the large lattice mismatch between the two domains to generate a compressive strain of the CdSe core and a strong piezoelectric potential along its c-axis. Efficient charge separation results in an indirect ground-state transition with a lifetime of several microseconds, almost one order of magnitude longer than any other $\mathrm{CdSe} / \mathrm{CdS}$ nanocrystal. Higher excited states recombine radiatively in the nanosecond time range, due to increasingly overlapping excited-state orbitals. $k \cdot p$ calculations confirm the importance of the anisotropic shape and crystal structure in the buildup of the piezoelectric potential. Strain engineering thus presents an efficient approach to highly tunable single- and multiexciton interactions, driven by a dedicated core/shell nanocrystal design.
\end{abstract}

\footnotetext{
${ }^{1}$ Nanochemistry Department, Istituto Italiano di Tecnologia, via Morego 30, IT-16163 Genova, Italy. ${ }^{2}$ Department of Physics, University of Genoa, via Dodecaneso 33, IT-16146 Genova, Italy. ${ }^{3}$ Departament de Quimica Fisica i Analitica, Universitat Jaume I, ES-12080 Castellón, Spain. ${ }^{4}$ Dipartimento di Scienza dei Materiali, Università degli Studi di Milano-Bicocca, via Cozzi 55, IT-20125 Milano, Italy. ${ }^{5}$ IBM Research-Zurich, Säumerstrasse 4, CH-8803 Rüschlikon, Switzerland. Correspondence and requests for materials should be addressed to J.P. (email: josep.planelles@uji.es) or to S.B. (email: brovelli@mater.unimib.it) or to I.M. (email: iwan.moreels@iit.it).
} 
G aining control over strain, through externally applied forces or via epitaxial growth of heterostructures with different lattice constants, is an important aspect of materials science. In particular, for optoelectronic and photonic applications, the addition of small distortions to the periodic lattice through inclusion of tensile or compressive strain shapes the band structure of semiconductors and the local band offset at the interface between different materials. Strain engineering has already found applications in diverse fields, with earlier examples showing, for instance, improved performance of silicon field-effect transistors via modified carrier mobilities ${ }^{1,2}$ and reduced lasing thresholds in strained InGaAs quantum wells ${ }^{3}$. Uniaxial strain is also predicted to induce an indirect-to-direct bandgap transition in germanium ${ }^{4}$, which could lead to efficient light emission from group-IV materials ${ }^{5}$. In epitaxial quantum dots, strain is used to switch between heavy and light holes in the ground-state exciton ${ }^{6}$, or to minimize the $\mu \mathrm{eV}$ fine-structure splitting between bright excitons ${ }^{7}$.

Colloidal nanocrystals (NCs) form a particular class of materials in which strain can be exploited to unprecedented levels. Due to the small dimensions of the template-free NCs $(<100 \mathrm{~nm})$, they can withstand significant elastic deformations ${ }^{8}$. In small NCs, the equilibrium lattice constant even differs from bulk, as demonstrated in $\mathrm{ZnS}(1 \% \text { contraction })^{9}$ or PbSe $(0.8 \%$ dilatation $)^{10}$. Hence, colloidal core/shell heteronanocrystals (heteroNCs) can be grown under large lattice mismatch conditions, leading to for instance high-quality $\mathrm{CdSe} / \mathrm{CdS}$ (4.4\% mismatch for zincblende, ZB, crystal structure), CdTe/ CdSe (6.4\%) and CdTe/ZnSe (13.4\%) NCs ${ }^{11-13}$. Furthermore, in several systems local band warping at the core/shell interface has been observed ${ }^{14-16}$. In CdTe/ZnSe heteroNCs, strain has been employed to invert the band offset from a type-I to a type-II heterostructure $^{11}$, and for CdSe/CdTe it has a strong influence on the carrier relaxation and recombination dynamics ${ }^{17-20}$. Nevertheless, in most applications interfacial strain is avoided, since it may also lead to the formation of interface defects that reduce the emission efficiency ${ }^{12,13}$.

Strain engineering can also be a means to construct a unique type of nanostructure, typically in NCs with a non-centrosymmetric crystal lattice. The absence of an inversion centre leads to a range of symmetry-dependent properties such as piezo- and pyroelectricity, chirality and circular dichroism ${ }^{21}$. Strain-induced piezoelectric fields have been demonstrated to lead to a significantly modified band structure and electron-hole overlap in $\mathrm{CdSe} / \mathrm{CdS}$ Stark superlattices ${ }^{22}$ and epitaxial III-nitride quantum $\operatorname{dots}^{23}$. This effect resulted from the wurtzite (WZ) crystal structure and from lattice mismatches of 2.5 and $4.2 \%$, and 4 and 3.9\% (ref. 24), along $a$ - and $c$-axes, respectively, that give rise to piezoelectric fields larger than $10^{6} \mathrm{~V} \mathrm{~cm}^{-1}$, an order of magnitude stronger than in ZB crystals $^{22,25}$. In colloidal NCs, it would imply a new direction in controlling the optoelectronic properties, in addition to quantum confinement via the NC size and shape, band structure engineering through the growth of heterostructures ${ }^{11-13}$ or controlled doping to induce highly localized electronic states ${ }^{26}$.

In this work, we demonstrate a direct control over the excitonic properties of NC quantum dots, through creation and manipulation of strong intra-particle piezoelectric fields, driven by the engineered interfacial strain in a new class of colloidal heterostructures. Using WZ rod-in-rod (RIR) CdSe/CdS NCs with minimum dimensions larger than the respective bulk Bohr radii of CdSe and CdS, 5.6 and $2.8 \mathrm{~nm}$, respectively, we exploit the strain-induced piezoelectric fields to create two spatially separated potential wells for electrons and holes at opposite sides of a $26 \mathrm{~nm}$ long CdSe nanorod. We hereby constructed a long-lived, indirect exciton with a lifetime of $4.4 \mu \mathrm{s}$, which is almost an order of magnitude longer than decay times previously reported for $\mathrm{CdSe} / \mathrm{CdS}$ heteroNCs ${ }^{27-29}$. In the multiexciton regime, such a band structure implies nearly unscreened repulsive Coulomb interactions between electrons and holes. Consequently, excited-state carriers still easily escape the shallow potential wells, leading to a large electron-hole wavefunction overlap for higher excited states with efficient, blue-shifted multiexciton emission and nanosecond recombination dynamics. Next to the unique approach to using internal electric fields to design the carrier potential wells, the relatively large dimensions, with strong electron-hole separation and associated microsecond exciton lifetimes, multiexciton blue shifts and reduced Auger recombination, give rise to a nanostructure that is optimally shaped for photonic applications ranging from gated fluorescence and exciton storage ${ }^{30}$ to single-exciton lasing ${ }^{31}$, via an exceptional degree of control over the charge carrier localization.

\section{Results}

RIR strain characterization. The synthesis of CdSe nanorods is adapted from the procedure described by Miszta et al. ${ }^{32}$, and yields NCs with sizes from $3 \mathrm{~nm}$ (diameter) by $9 \mathrm{~nm}$ (length), up to $11 \mathrm{~nm}$ by $26 \mathrm{~nm}$, that is, beyond the strong confinement regime (Table 1; Fig. 1a; Supplementary Fig. 1). To obtain a sizable CdS shell around such large CdSe cores, we introduced $\mathrm{CdCl}_{2}$ in the synthesis ${ }^{32,33}$, which activates the shell growth via otherwise unreactive facets in accordance with the synthesis of CdSe/CdS octapods $^{33}$. This avoids homogeneous nucleation of CdS clusters and leads to an overall $30 \mathrm{~nm}$ by $85 \mathrm{~nm}$ heteroNC in typically 10 min (Table 1; Supplementary Figs 1 and 2). Structural characterization by X-ray diffractometry (XRD) confirms the growth of WZ core/shell RIRs (Fig. 1b). XRD patterns of both CdSe seeds and CdSe/CdS heteroNCs display sharper [0002] diffraction peaks around $25^{\circ} 2 \theta$, confirming that the $c$-axis corresponds to the NC long axis. As evidenced by high-angle annular dark-field scanning transmission electron microscopy (HAADF-STEM) and elemental analysis with energy-dispersive X-ray spectroscopy (EDS) line scans (Fig. 1c), the growth in both directions along the $c$-axis also yields more symmetric core/shell NCs, with the CdSe core nanorod located at the centre of the heterostructure. This is in contrast with the seeded growth of CdSe/CdS dot-in-rods (DIRs) ${ }^{34}$, and previous RIR syntheses ${ }^{35}$, where the core is typically located towards one end of the heteroNC. High-resolution TEM (HRTEM) shows few stacking faults within the heteroNCs and confirms an epitaxial shell growth according to the following symmetry relationships: $\mathrm{CdS}[0002] / / \mathrm{CdSe}[0002]$ and $\mathrm{CdS}[10 \overline{10}] / / \mathrm{CdSe}[10 \overline{1} 0]$ (Fig. 1d; Supplementary Fig. 3; Supplementary Note 1). Raman spectroscopy revealed that, despite the fast growth of the shell,

\begin{tabular}{|c|c|c|c|c|}
\hline Sample & $\begin{array}{c}\text { Diameter } \\
(\mathbf{n m})\end{array}$ & $\begin{array}{c}\text { Length } \\
(\mathbf{n m})\end{array}$ & $\begin{array}{c}\text { Emission } \\
(\mathbf{n m})\end{array}$ & $\begin{array}{c}\text { Lifetime } \\
\text { (ns) }\end{array}$ \\
\hline Core sample I & 3.2 & 8.9 & 560 & 14 \\
\hline RIR sample I & 9.7 & 52.0 & 643 & 102 \\
\hline Core sample II & 4.8 & 15.0 & 633 & 15 \\
\hline RIR sample II & 9.8 & 43.8 & 670 & 853 \\
\hline Core sample III & 10.8 & 25.6 & 695 & 24 \\
\hline RIR sample III & 28.9 & 84.8 & 733 & 4,450 \\
\hline
\end{tabular}



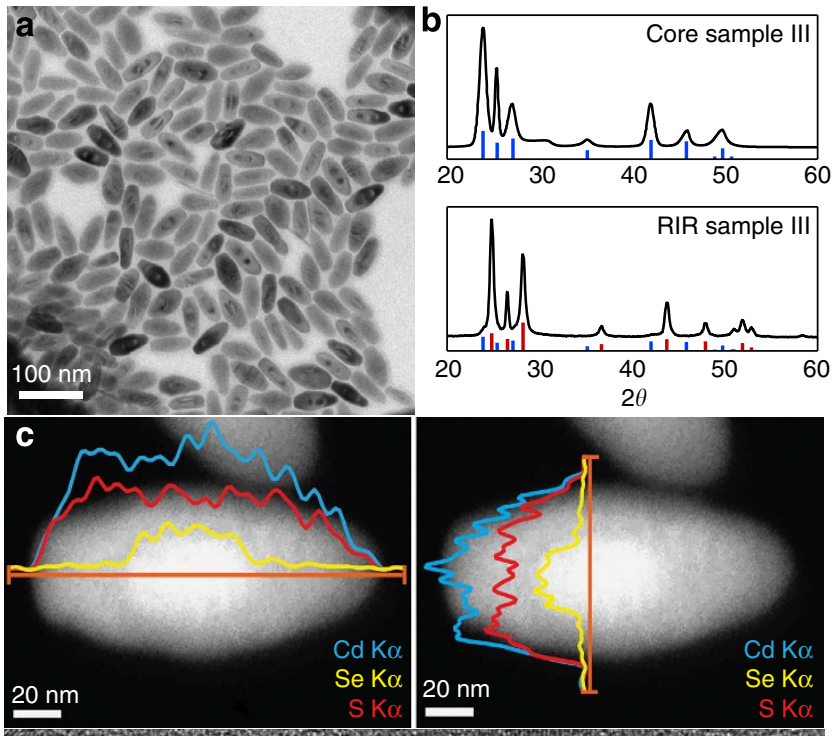

di:

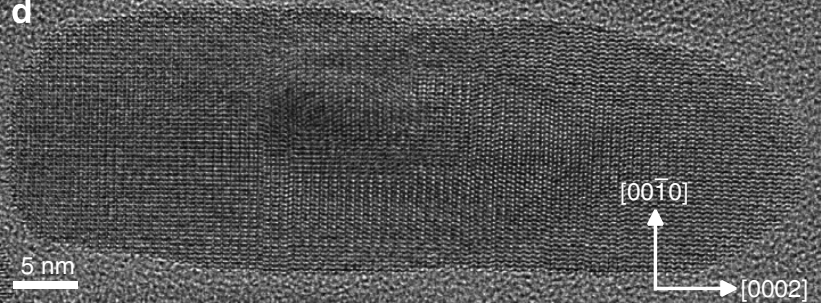

Figure 1 | Structural characterization of the RIRs. (a) Overview TEM of $\mathrm{CdSe} / \mathrm{CdS}$ RIR sample III. The dark and light areas around the CdSe cores are due to diffraction contrast generated by the local distortion of the lattice near the core/shell interface. (b) XRD patterns of core CdSe nanorods and corresponding core/shell RIRs (sample III). Vertical lines indicate the bulk WZ CdSe (blue) and CdS (red) diffraction angles. (c) HAADF-STEM image of single RIRs, with projected composition determined from the EDS profile (along orange line) of $\mathrm{Cd} \mathrm{K} \alpha, \mathrm{S} \mathrm{K} \alpha$ and Se K $\alpha$ signals. (d) HRTEM image of a single RIR (Sample III) showing epitaxial growth of $\mathrm{CdSe} / \mathrm{CdS}$ core/shell heterostructures.

the RIR NCs exhibit a $\mathrm{CdSe}_{x} \mathrm{~S}_{1-x}$ alloy region at the CdSe/CdS interface (Supplementary Fig. 4; Supplementary Table 1; Supplementary Note 2), likely due to the $380^{\circ} \mathrm{C}$ growth temperature used.

Several RIR NCs in the bright-field TEM image (Fig. 1a) show a particular contrast, occurring either near the tips of the CdSe core or along its sides. This can be attributed to a local deformation of the crystal lattice near the CdSe/CdS interface (Ashby-Brown diffraction contrast ${ }^{36-38}$ ), and already gives a first indication of significant strain in our NCs. To further evaluate the deformation, we calculated the strain fields of the HRTEM images (Fig. 2d) using the peak pairs analysis ${ }^{39}$. The coordinates of the atomic $u_{i j}$ displacements were chosen such that the $z$-axis of the strain maps was parallel to the crystallographic $c$-axis, and the $y$ axis corresponded to the $[10 \overline{1} 0]$ axis. Figure 2 a shows the resulting mean dilatation strain map extrapolated from the HRTEM image reported in Fig. 1c. It reveals a significant dilatation of the CdS lattice near the CdSe core (central green region), with respect to the CdS crystal lattice at the far ends of the heteroNC. Importantly, due to the central position of the CdSe core nanorod and the epitaxial growth of the CdS shell, the lattice deformation can develop symmetrically at both sides of the heterostructure. We quantified the (projected) area with a uniform crystal lattice (Supplementary Table 2), and found that the diameter obtained via HAADF-STEM and EDS line scans
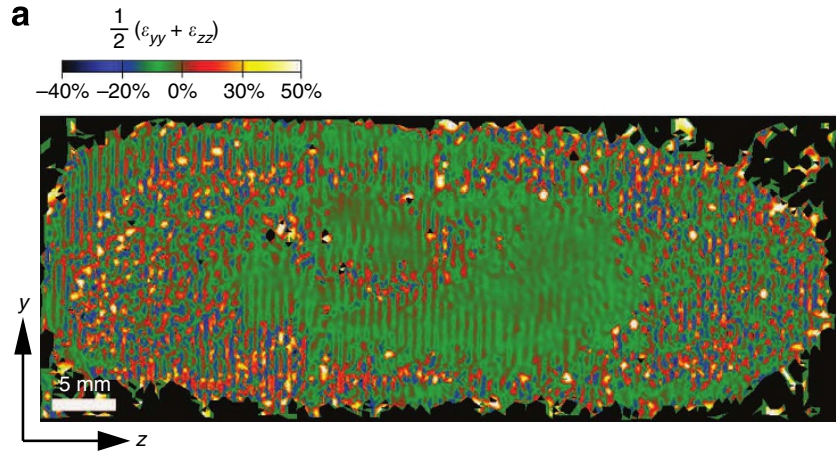

b
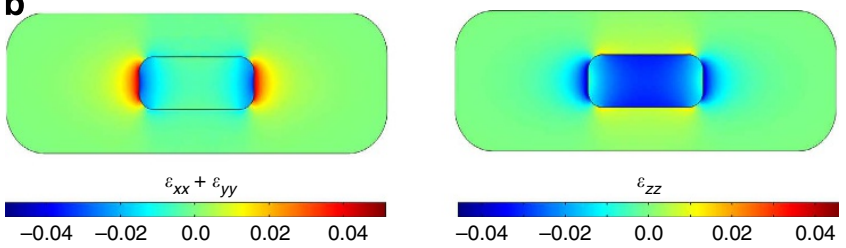

Figure 2 | Experimental and theoretical strain analysis. (a) Mean dilation strain map of the RIR NC shown in Fig. 1c (scale ranges from $-40 \%$ to $50 \%$ for an optimum contrast). Few stacking faults and dislocations locally distort the strain field, yet we observe a region of homogeneous strain (green) of about $15 \times 30 \mathrm{~nm}$, clearly larger than the $11 \times 26 \mathrm{~nm}$ core CdSe nanorod. The $z$ - and $y$-tensor axes correspond to [0002] and [1010] crystallographic axes, respectively. (b) Calculated lateral (left panel) and c-axis (right panel) strain tensor components, showing the compression of the CdSe core and dilatation of the CdS shell at the CdSe/ CdS interface.

is $1.4 \mathrm{~nm}$ smaller than the strain profile. A more pronounced extension of the core structure is observed in the axial direction, by $5.0 \mathrm{~nm}$ on either side. This can be ascribed to a significant lattice deformation inside the CdSe core and at the core/shell interface, which extends the strain field into the CdS shell.

Experimental data are supported by theoretical calculations of the strain. Figure $2 \mathrm{~b}$ shows the lateral $\left(\varepsilon_{x x}+\varepsilon_{y y}\right)$ and axial $\left(\varepsilon_{z z}\right)$ strain tensor components of a large RIR, defined with respect to the natural lattice constants of the CdSe core and the CdS shell in each region. The strong compressive strain of the core along the $c$-axis $\left(\varepsilon_{z z}<0\right)$, along with the tensile strain of the shell surrounding the lateral sides $\left(\varepsilon_{z z}>0\right)$, show that both materials tend to minimize the structural mismatch on the lateral interface by deforming the axial lattice constants. The same occurs with the lateral strain near the CdSe core tips, where the core is again compressed $\left(\varepsilon_{x x}+\varepsilon_{y y}<0\right)$ and the shell is dilated $\left(\varepsilon_{x x}+\varepsilon_{y y}>0\right)$. The extent of compression and dilatation is in line with the expansion of the core crystal lattice into the shell, as observed by HRTEM strain analysis. More importantly, the elastic behaviour of the RIR lattice implies that the compression/ dilatation of core and shell is followed by an opposite response in the orthogonal directions. This leads to a very weak lateral strain on the long sides of the rod (core and shell roughly preserve their natural in-plane constants) and a strongly compressive axial strain of the CdS lattice near the tips. The severe anisotropy of the strain in these structures gives rise to strong polarization along the axial direction, which translates into the intense piezoelectric field. The large thickness of the CdS shell leads to a situation where the outer CdS monolayers are nearly undeformed. As a result, slightly different shell thickness within this size regime should have no significant influence on the strain ${ }^{40}$. 
a

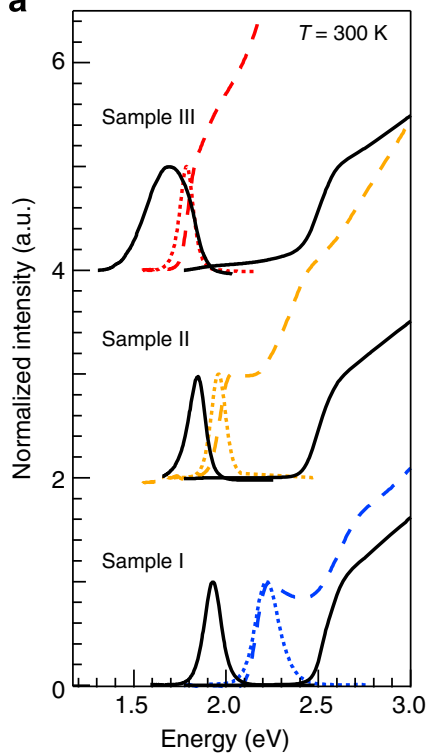

b

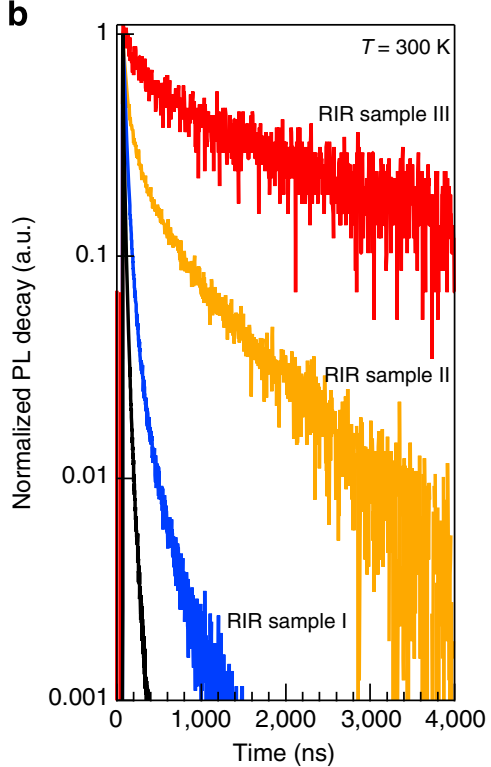

c
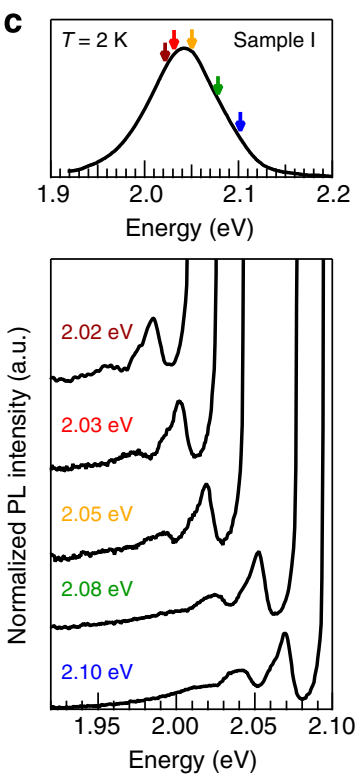

d
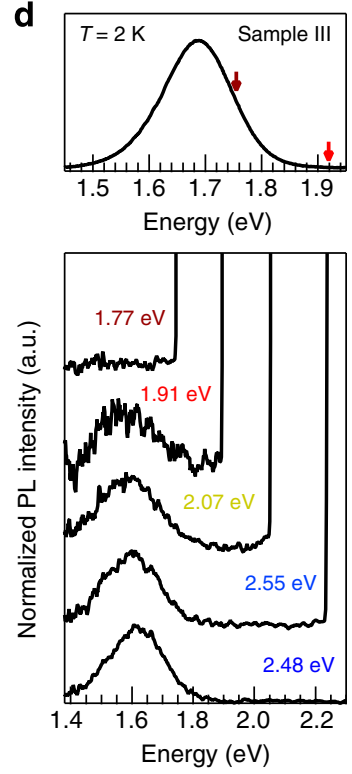

Figure 3 | Optical confirmation of the indirect exciton formation. (a) Room temperature absorbance spectra of CdSe core nanorods (dashed lines) and $\mathrm{CdSe} / \mathrm{CdS}$ core/shell RIRs (full lines), with corresponding PL spectra of core (dotted lines) and core/shell RIRs (full lines). Note that in RIR sample III, Rayleigh scattering is observed, possibly due to the large RIR volume. (b) Time-resolved PL decay traces of CdSe nanorods (black), and RIR samples I, II and III. We observe a lifetime up to $4.4 \mu \mathrm{s}$, for RIR sample III. (c,d) Fluorescence line-narrowing (FLN) spectra at $2 \mathrm{~K}$ of quasi-type-II heteroNCs (RIR sample I, c) and type-II heteroNCs (RIR sample III, d). The full PL spectrum under non-resonant excitation at $3.1 \mathrm{eV}$ is plotted on top for reference, with arrows indicating the excitation wavelength.

Band structure and optical properties. With experimental and theoretical verification of the presence of the strain in our WZ RIR heteroNCs, we proceeded with measuring its influence on the single-exciton and multiexciton emission properties. In Fig. 3, we report the optical absorption and photoluminescence (PL) spectra of three representative CdSe/CdS RIRs with increasing core size (PL excitation spectra are reported in Supplementary Fig. 5). Interestingly, upon growing the $\mathrm{CdS}$ shell, we observed a significant red shift of the PL spectrum even for the largest RIRs (RIR sample III, Fig. 3a), whose PL peaked at $1.69 \mathrm{eV}$. This emission energy is well below the bandgap energy of bulk CdSe $\left(E_{\mathrm{g}}=1.74 \mathrm{eV}\right)$ and thus provides a first indication of an indirect exciton transition. Time-resolved PL decay traces provide further evidence of a significantly reduced electron-hole overlap in strain-engineered RIR NCs with respect to conventional unstrained materials (Fig. 3b). Remarkably, we obtained PL lifetimes varying from $\tau=100 \mathrm{~ns}$, for the RIR with the smallest core (RIR sample I), up to $\tau=4.4 \mu$ s for the largest RIR (RIR sample III). This value is over two orders of magnitude longer than the emission lifetime of the corresponding core-only CdSe nanorods (15-20 ns, Table 1), and surpasses also any precedent literature data on CdSe/CdS giant-shell DIDs with $\mathrm{ZB}^{28,29}$ and WZ cores $^{27}$, which exhibit lifetimes up to $650 \mathrm{~ns}$, and even intrinsic type-II CdSe/CdTe quantum dots ${ }^{41}$, nanorods ${ }^{42}$ and two-dimensional heteroplates, with lifetimes reaching $1.8 \mu \mathrm{s}^{43}$. Note that the long lifetime here is not due to trap state emission, as we observed a continuous PL shift and increase in PL lifetime upon shell growth, in line with a progressive reduction of the electron-hole overlap (Supplementary Fig. 6). In addition, similar to spherical giant-shell NCs ${ }^{12,13,27,28,44}$, due to the thick CdS shell the electron and hole wavefunctions are efficiently decoupled from CdS surface traps, leading to a consistently high PL quantum efficiency of 15,10 and $23 \%$ for the samples I, II and III, respectively.

To further confirm the transition from direct to indirect ground-state excitons in strain-engineered RIRs, we performed fluorescence line-narrowing (FLN) experiments at $2 \mathrm{~K}$. Here, samples are excited by a narrow-band continuous-wave light source tuned to the red side of the band-edge absorption, which allows photoexciting a small fraction of the NC ensemble. As a result, we can resolve the spectroscopic features of longitudinal optical (LO) phonon-assisted transitions ${ }^{45,46}$. Resonant excitation of RIR sample I yields a structured FLN spectrum showing the typical vibronic progression associated with $\mathrm{LO}$ phonon modes of $\mathrm{CdSe} \quad\left(\mathrm{LO}_{\mathrm{CdSe}}=25 \mathrm{meV}\right)$ and $\mathrm{CdS}\left(\mathrm{LO}_{\mathrm{CdS}}=35 \mathrm{meV}\right)$, and respective overtones (Fig. 3c; Supplementary Fig. 7). This indicates a so-called quasi-type-II band offset, characterized by a partial delocalization of the electron wavefunction over both the CdSe core and the CdS shell. On the other hand, resonant excitation of RIR sample III at $1.77 \mathrm{eV}$ yields no measurable emission even for very long accumulation times (as long as $1 \mathrm{~h}$ ). A weak PL is first observed by exciting the RIRs at least $140 \mathrm{meV}$ above the energy of the PL maximum at $1.9 \mathrm{eV}$, at which point a featureless PL spectrum appears due to the excitation of the entire RIR ensemble (Fig. 3d). This clearly demonstrates that strained RIRs possess a large intrinsic Stokes shift that, together with the microsecond-long PL lifetime, is an unambiguous signature of indirect exciton formation.

The observed optical properties of RIRs are markedly different from those of CdSe/CdS heteroNCs with a spherical core, where a transition to type-II behaviour typically occurs only in the limit of strong confinement and core diameters below $3 \mathrm{~nm}^{47,48}$. This strongly suggests that the strain must have a profound influence on the electronic structure of our elongated heteroNCs that have considerably bigger core diameters. Indeed, $k \cdot p$ calculations show that the hydrostatic strain potential already raises the CdSe conduction band, but a type-I band offset is still preserved (Supplementary Fig. 8). What truly differentiates the RIRs from previously reported DIDs and DIRs is the strain-induced piezoelectricity in combination with the elongated shape of the WZ CdSe core. In the largest RIR, the $0.15 \mathrm{MV} \mathrm{cm}^{-1}$ piezoelectric field builds up a potential difference of $0.45 \mathrm{eV}$ across the long axis of the CdSe core (Fig. 4a,b; Supplementary Figs 9 and 10). This yields a local conduction band minimum and 
a

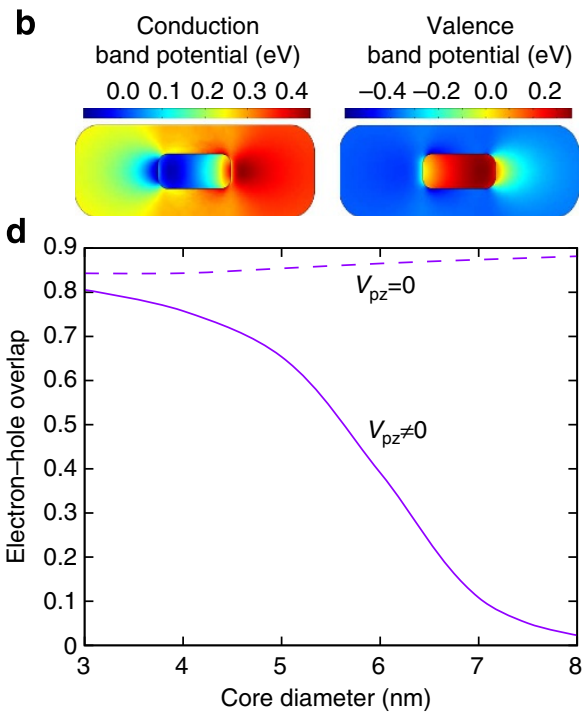

Figure 4 | $\boldsymbol{k} \cdot \boldsymbol{p}$ calculations of the CdSe/CdS band structure. (a) Plot of the overall band structure along the long axis of the RIR sample III. The potential wells are strongly asymmetric, with a valence band maximum and conduction band minimum at opposite sides of the core CdSe nanorod. The indirect transition also has an energy below the bulk CdSe bandgap. (b) Corresponding two-dimensional plots. (c) Electron (left) and hole (right) wavefunctions are well separated, confirming the indirect exciton ground state. (d) The electron-hole overlap for RIRs of increasing size rapidly decreases when piezoelectricity is taken into account (solid line), while it otherwise remains above $80 \%$ (dashed line). The core has an aspect ratio of 1:3 and the shell is twice as large.

valence band maximum at opposite sides of the CdSe core nanorod, with electrons and holes that are almost completely separated and confined to their individual, $0.22 \mathrm{eV}$ shallow potential wells (Fig. 4c). Considering an initial conduction band offset of $0.32 \mathrm{eV}$ (ref. 49), we estimated a reduction of the electron-hole wavefunction overlap by two orders of magnitude after shell growth, consistent with the strong increase in lifetime observed experimentally $(4.4 \mu$ s for RIR sample III, compared with $24 \mathrm{~ns}$ observed for the corresponding core-only CdSe nanorods). The overlap can be controlled by the rod length, eventually returning to a reduced piezoelectric field and more symmetric band structure in RIR sample I, where the electronhole overlap is again mostly dominated by the band offset at the $\mathrm{CdSe} / \mathrm{CdS}$ interface (Supplementary Fig. 11). The critical role of piezoelectricity in determining the electron-hole overlap of single excitons in RIR is further evidenced in Fig. 4d, which compares the calculated overlap for a series of RIRs with increasing size (aspect ratio: 1:3). A pronounced damping of the electron-hole overlap takes place when piezoelectricity is considered (solid line), which is otherwise missing (dashed line). Similar results are obtained with different conduction band offsets (Supplementary Table 3), which indicates that the role of piezoelectricity is robust within the uncertainty range of model parameters. It clearly shows that the piezoelectric effect induces carrier (de-)localization beyond what can be reached with quantum confinement or interfacial hydrostatic strain alone. These heteroNCs should therefore also be highly sensitive to external pressure or voltage, providing an interesting prospect as stress gauge $^{50}$ or charge sensor ${ }^{51}$.

Multiexciton emission. In the light of the remarkable effects of strain on the single-exciton behaviour, we also expect substantial differences between the multiexciton (MX) features of strainengineered RIRs with respect to conventional, non-strained materials. We prepared thin films of the RIRs and cooled the sample to $4 \mathrm{~K}$ (Supplementary Fig. 12) to measure the fluence-dependent emission spectra. The PL spectrum of RIR sample I displays a weak, $28 \mathrm{meV}$ blue-shifted peak at high excitation fluence (Supplementary Fig. 13), consistent with biexciton emission observed on DIRs ${ }^{48}$ with a small CdSe core. RIR sample III on the other hand exhibits a far larger, $140 \mathrm{meV}$ blue-shifted peak that clearly dominates the emission spectrum at high fluence (Fig. 5a). The linear growth of the PL intensity with increasing fluence (Fig. 5b) implies a strongly reduced Auger recombination in these heteroNCs, similar to giant-shell DIDs ${ }^{46}$, and is possibly efficiently suppressed by the elongated CdSe core ${ }^{52}$ and smoothed wavefunctions due to interface alloying $^{53-55}$ (Supplementary Fig. 4). The emission dynamics at high fluence is multiexponential with nanosecond lifetime components of 1.9 and $20 \mathrm{~ns}$, respectively (Fig. 5c,d), significantly longer than what is expected from volume scaling alone (300-400 $\mathrm{ps}^{46}$, taking a volume of $2,345 \mathrm{~nm}^{3}$ corresponding to the CdSe core). Considering the suppressed Auger recombination, the MX recombination must therefore involve electrons and holes with a significantly larger wavefunction overlap with respect to single-exciton carriers. $k \cdot p$ calculations support this view. Unique to this system, the shallow nature of piezoelectric potentials (Fig. 4a) yields excited-state orbitals that are increasingly delocalized over the CdSe core (Fig. 5e,f). Since the electronic configuration of multiexcitons is constructed from such orbitals, their electron-hole overlap will be increasingly large. In contrast to the indirect ground-state transition, we can therefore expect a substantially enhanced multiexciton radiative rate. Considering both the $140 \mathrm{meV} \mathrm{MX}$ blue shift and the three orders or magnitude difference between X and MX lifetimes, the results thus suggest that strain-engineered colloidal NCs open up new pathways towards single-exciton lasers ${ }^{31}$, due to the wellseparated single-exciton emission, or even novel biexciton lasing concepts that suppress Auger recombination by shape-control ${ }^{56}$.

\section{Discussion}

With large WZ CdSe rods embedded in giant CdS shells, we have synthesized a unique colloidal system with a band structure determined by piezoelectric fields. The resulting indirect excitons 


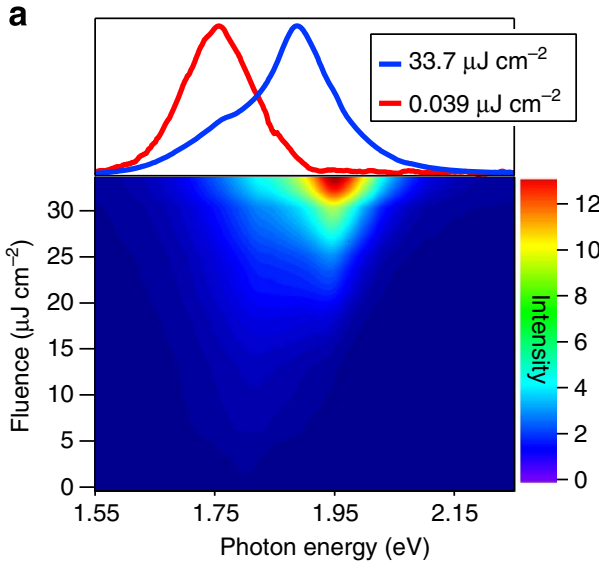

b

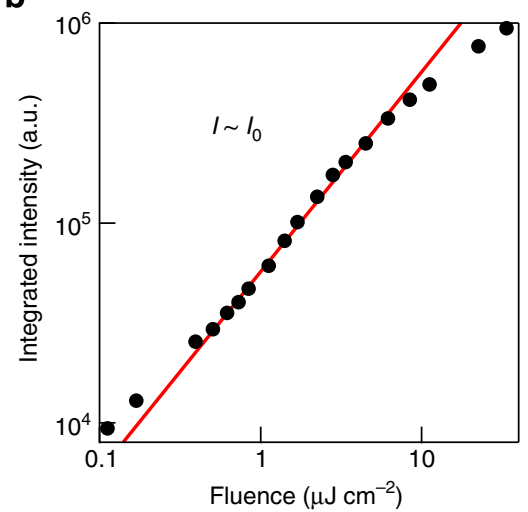

C

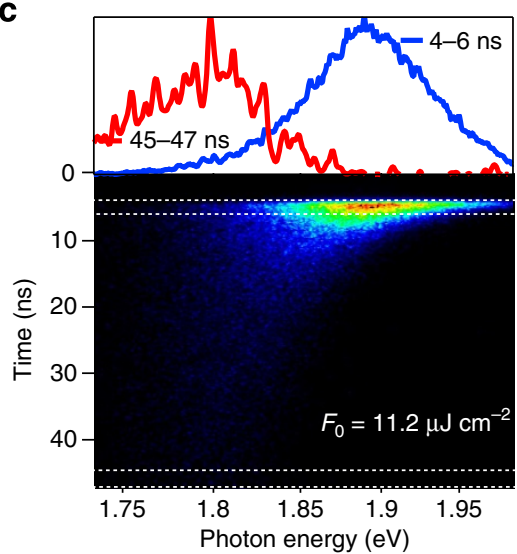

d

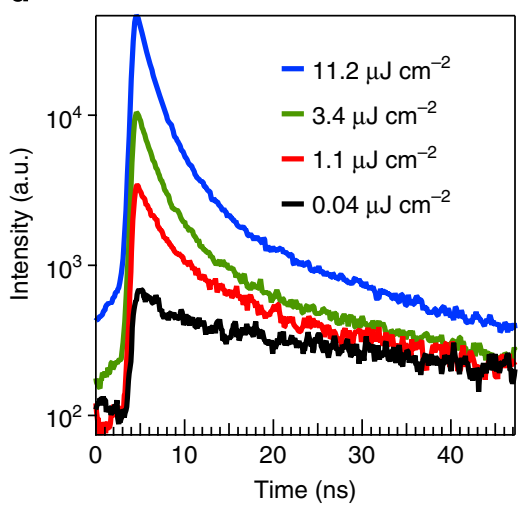

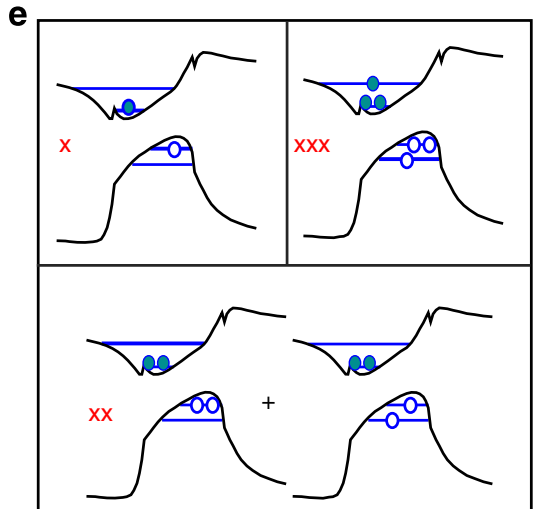

f CdS CdSe CdS

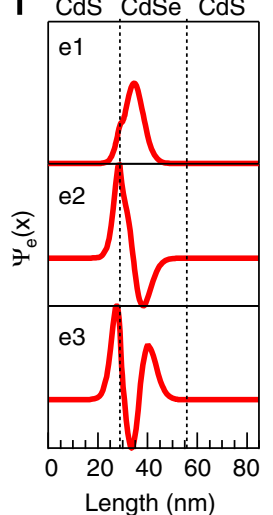

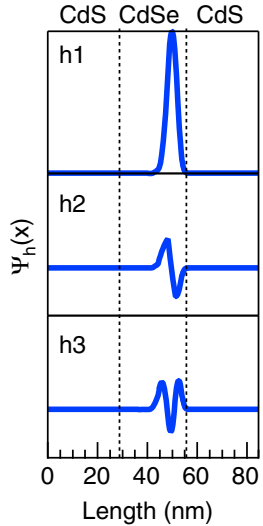

Figure 5 | Multiexciton emission in RIRs. (a) Fluence-dependent PL spectra of RIR sample III. At high fluence, a 140 meV blue shift of the emission is observed. (b) The PL intensity increases linearly with fluence up to $10 \mu \mathrm{Jm}^{-2}$, suggesting strongly suppressed Auger recombination. (c) Streak camera image at a fluence of $11.2 \mu \mathrm{cm}^{-2}$, confirming the blue-shifted $\mathrm{MX}$ emission. The emission at $45 \mathrm{~ns}$ is shown for comparison; however, it does not yet correspond to the pure single-exciton $\mathrm{PL}$, which should peak at $1.75 \mathrm{eV}$. (d) The spectrally integrated decay is multiexpontential, with lifetimes of 1.9 and $20 \mathrm{~ns}$, respectively. Note that MX emission can already be discerned below $1 \mu \mathrm{J} \mathrm{cm}{ }^{-2}$, while the linear increase of the $\mathrm{PL}$ persists up to $10 \mu \mathrm{J} \mathrm{cm}^{-2}$ (see $\mathbf{b}$ ). (e) Sketch of the main electronic configurations of exciton, biexciton and triexciton ground states. The occupation of higher lying orbitals, whose piezoelectric confinement is weaker, enables stronger electron-hole overlap for bi- and triexcitons. (f) Electron and hole wavefunctions for the three lowest electron and hole orbitals in RIR sample III, showing an increasing shift towards the core centre.

have lifetimes up to $4.4 \mu \mathrm{s}$, with an electron-hole delocalization that can be carefully controlled by the material parameters. Next to a respectable PL quantum efficiency of 10-23\% (considering the typeII configuration), these materials also exhibit efficient multiexciton emission and suppressed Auger recombination. This may not only lead to deepened insights into the excited-state carrier dynamics in colloidal nanocrystals, but may also open the way to further improvements in quantum dot-based energy harvesting ${ }^{57-59}$ or optoelectronic $^{31,60}$ applications. In addition, the exceptionally long lifetimes could find, among others, application in dye-sensitized solar cells ${ }^{61}$ or exciton storage devices ${ }^{30}$.

\section{Methods}

Materials. Tri- $n$-octylphosphine oxide (TOPO, 99\%), tri- $n$-octylphosphine (TOP, 97\%) and selenium (Se, 99.99\%) were purchased from Strem Chemicals. Cadmium oxide (CdO, $99.99 \%)$, cadmium chloride $\left(\mathrm{CdCl}_{2}, 99.99 \%\right)$, sulfur (S, $\left.99.98 \%\right)$, ethanol, toluene and chloroform were purchased from Sigma-Aldrich. $\mathrm{N}$-octadecylphosphonic acid (ODPA) and $n$-hexylphosphonic acid (HPA) were purchased from Polycarbon Industries.

Synthesis of CdSe cores. CdSe seeds were synthesized using a procedure adapted from Miszta et al. ${ }^{32}$ For example, to obtain the largest nanorods with a diameter of $10.6 \mathrm{~nm}$ and length of $25.6 \mathrm{~nm}$ (core of RIR sample III), $3 \mathrm{~g}$ of TOPO, $50 \mathrm{mg}$ of CdO, $80 \mathrm{mg}$ of HPA and $260 \mathrm{mg}$ of ODPA have been added into a flask and degassed for $1 \mathrm{~h}$ at $150^{\circ} \mathrm{C}$. Then the temperature was increased to $380^{\circ} \mathrm{C}$ under an argon flow, and $2.6 \mathrm{ml}$ of TOP was injected. When the temperature of $380^{\circ} \mathrm{C}$ was re-established, $1 \mathrm{ml}$ of a $0.15 \mathrm{M}$ TOPSe solution was injected and the nanorods were grown for $10 \mathrm{~min}$. To synthesize smaller nanorods, the TOPSe concentration, the precursor and ligand concentrations and reaction temperature and time were changed (Supplementary Note 3).

Synthesis of CdSe/CdS RIRs. In a $50 \mathrm{ml}$ round-bottom flask, $3 \mathrm{~g}$ of TOPO, $50 \mathrm{mg}$ of $\mathrm{CdO}, 6 \mathrm{mg}$ of $\mathrm{CdCl}_{2}, 80 \mathrm{mg}$ of HPA and $260 \mathrm{mg}$ of ODPA were degassed at $150^{\circ} \mathrm{C}$ under vacuum for $1 \mathrm{~h}^{32}$. Then, under argon flow the temperature was further raised to $380^{\circ} \mathrm{C}$, and $2.6 \mathrm{ml}$ of TOP was injected. After the temperature of $380^{\circ} \mathrm{C}$ was re-established, a mixture of $0.5 \mathrm{~g}$ of TOPS (from a preheated, supersatured TOPS stock solution containing $96 \mathrm{mg}$ of $\mathrm{S}$ in $1 \mathrm{ml}$ of TOP) and $150 \mu \mathrm{l}$ of a $3 \mu \mathrm{M}$ solution of CdSe nanorods in TOP were added. The CdS shell was grown for $10 \mathrm{~min}$. Next, the solution was rapidly cooled to room temperature and $10 \mathrm{ml}$ of toluene was added. The RIRs were purified by adding $5 \mathrm{ml}$ of ethanol and centrifuging them at 3,000 r.p.m. for $5 \mathrm{~min}$, after which they were redispersed in chloroform. This was repeated three times, and the RIRs were finally dispersed in $5 \mathrm{ml}$ of chloroform.

Structural characterization. X-ray diffractograms were measured with a Rigaku SmartLab $9 \mathrm{~kW}$ diffractometer operated at $40 \mathrm{kV}$. The XRD samples were obtained by dropcasting the NCs onto a miscut silicon substrate. Bright-field TEM measurements were carried out on a JEOL-1100 TEM operating at an acceleration voltage of $100 \mathrm{kV}$. HRTEM was performed with a JEOL JEM-2200FS microscope equipped with a field emission gun working at an accelerating voltage of $200 \mathrm{kV}$, a CEOS spherical aberration corrector of the objective lens, which enables a spatial resolution of $0.9 \AA$, and an in-column Omega filter. HAADF-STEM images were collected using a spot size of $0.7 \mathrm{~nm}$. The chemical composition of the RIRs was determined by EDS line scan analysis performed in HAADF-STEM mode, using a Bruker Quantax 400 system with a $60 \mathrm{~mm}^{2}$ XFlash 6T silicon drift detector according to the Cliff-Lorimer method. HRTEM images were also used for 
strain analysis with the peak pairs analysis method ${ }^{39}$. The structural displacements $u_{i j}$ of the atomic columns were used to calculate the (projected) $y z$ strain tensor components, $\varepsilon_{z z}=\frac{\vartheta u_{z}}{\partial z}, \varepsilon_{y y}=\frac{\vartheta u_{y}}{\partial y}, \varepsilon_{z y}=\frac{1}{2}\left(\frac{\vartheta u_{z}}{\partial y}+\frac{\vartheta u_{y}}{\partial z}\right)$, considering as the lattice basis vectors the [0002] and [1010] structural reflections of the WZ lattice. The mean dilatation map was subsequently calculated from the mean value of the inplane tensor $\Delta_{y z}=\frac{1}{2}\left(\varepsilon_{y y}+\varepsilon_{z z}\right)$.

Raman spectroscopy. The NCs were dropcast on a glass substrate forming a closepacked film. Raman spectra were collected using a Renishaw InVia MicroRaman spectrometer, exciting the samples with a $100 \mathrm{~mW}$ diode laser at $\lambda=532 \mathrm{~nm}$, using a $\times 50$ magnification microscope objective, with integration times up to $30 \mathrm{~s}$.

Single-exciton PL measurements. PL measurements were performed using an Edinburgh Instruments FLS920 spectrofluorometer. Samples were excited at $400 \mathrm{~nm}$ with a Xenon lamp for the steady-state measurements, and at $405 \mathrm{~nm}$ with a pulsed laser (50 ps pulse duration) for the time-resolved traces. Typically, a region of $10 \mathrm{~nm}$ around the peak maximum was selected for the decay measurements. Quantum efficiencies were determined with an integrating sphere, exciting the RIR sample I and II at $400 \mathrm{~nm}$, and sample III at $450 \mathrm{~nm}$. The optical density was tuned to 0.1 at the excitation wavelength.

Fluorescence line-narrowing spectroscopy. For FLN measurements, a spectrally narrow photoexcitation source $(<0.2 \mathrm{~nm}$ full width at half maximum) was produced by filtering the output of a $150 \mathrm{~W}$ Xenon lamp with a $1 / 3 \mathrm{~m}$ double-grating monochromator. The emitted PL photons were collected, fibre-coupled into a $1 / 2 \mathrm{~m}$ spectrograph and detected with a nitrogen-cooled charged-coupled device. All measurements were performed at $2 \mathrm{~K}$.

Multiexciton PL spectroscopy. RIR films were deposited on sapphire substrates and cooled to $4 \mathrm{~K}$ in a closed-cycle helium cryostat (Advanced Research Systems). To excite the samples, a $70 \mathrm{fs}, 1 \mathrm{kHz}$ Ti:sapphire amplified laser was frequency doubled to an excitation wavelength of $405 \mathrm{~nm}$ and reduced to a $2 \mathrm{~mm}$ (diameter) excitation spot. The PL was relayed to an Avantes spectrophotometer for steadystate measurements, and to a Hamamatsu Photonics streak camera to measure the time-resolved spectral decay.

$\boldsymbol{k} \cdot \boldsymbol{p}$ Theory. Experimental data were compared with theoretical strain maps that were calculated in the continuous medium model by minimizing the elastic energy. The boundary conditions are zero normal stress for the free surface. Details on the underlying theory are given by Rajadell et al. ${ }^{40}$ The strain-induced piezoelectric polarization, which is linearly related to strain, is calculated by $P_{i}^{\text {strain }}=\sum_{k} e_{i k} \varepsilon_{k k}$. The determination of $\varepsilon_{i j}(\boldsymbol{r})$ is performed using the multiphysics mode of Comsol 4.2 software. Electrons $(j=e)$ and heavy-hole $(j=h h)$ states of the ground-state exciton are calculated from the Hamiltonian $H_{j}=H_{k}^{j}+V_{s}^{j}+V_{\mathrm{pz}}^{j}+V_{\mathrm{qc}}^{j}+V_{\mathrm{eh}}^{j}$, where $H_{k}^{j}$ and $V_{s}^{j}$ are the single-band effective mass kinetic energy and strain potential, $V_{\mathrm{pz}}^{j}$ is the piezoelectric potential, $V_{\mathrm{qc}}^{j}$ the quantum confinement due to the different band offset of core, shell and surrounding environment materials and $V_{\mathrm{eh}}^{j}$ the electrostatic potential created by the opposite sign carrier, which takes into account the dielectric mismatch with the dielectric surroundings of the RIR. Converged interacting electron and hole states are obtained by iterative resolution of the Schrödinger-Poisson equation (see Supplementary Table 4 for the material parameters)

\section{References}

1. Welser, J., Hoyt, J. L. \& Gibbons, J. F. Electron mobility enhancement in strained-Si n-type metal-oxide-semiconductor field-effect transistors. IEEE Electron Device Lett. 15, 100-102 (1994).

2. Abstreiter, G., Brugger, H., Wolf, T., Jorke, H. \& Herzog, H. J. Strain-induced two-dimensional electron gas in selectively doped Si/SixGe1-x superlattices. Phys. Rev. Lett. 54, 2441-2444 (1985).

3. Coleman, J. J. Strained layer InGaAs quantum-well heterostructure lasers. IEEE J. Sel. Top. Quantum Electron. 6, 1008-1013 (2000).

4. Zhang, F., Crespi, V. H. \& Zhang, P. Prediction that uniaxial tension along $\langle 111\rangle$ produces a direct band gap in germanium. Phys. Rev. Lett. 102, 156401 (2009).

5. Sánchez-Pérez, J. R. et al. Direct-bandgap light-emitting germanium in tensilely strained nanomembranes. Proc. Natl Acad. Sci. USA 108, 18893-18898 (2011).

6. Huo, Y. H. et al. A light-hole exciton in a quantum dot. Nat. Phys. 10, 46-51 (2014).

7. Tartakovskii, A. I. et al. Effect of thermal annealing and strain engineering on the fine structure of quantum dot excitons. Phys. Rev. B 70, 193303 (2004).

8. Shan, Z. W. et al. Ultrahigh stress and strain in hierarchically structured hollow nanoparticles. Nat. Mater. 7, 947-952 (2008).

9. Gilbert, B., Huang, F., Zhang, H., Waychunas, G. A. \& Banfield, J. F. Nanoparticles: strained and stiff. Science 305, 651-654 (2004).
10. Petkov, V., Moreels, I., Hens, Z. \& Ren, Y. PbSe quantum dots: finite, offstoichiometric, and structurally distorted. Phys. Rev. B 81, 241304 (2010).

11. Smith, A. M., Mohs, A. M. \& Nie, S. Tuning the optical and electronic properties of colloidal nanocrystals by lattice strain. Nat. Nanotechnol. 4, 56-63 (2009).

12. Mahler, B. et al. Towards non-blinking colloidal quantum dots. Nat. Mater. 7, 659-664 (2008).

13. Chen, Y. et al. 'Giant' multishell CdSe nanocrystal quantum dots with suppressed blinking. J. Am. Chem. Soc. 130, 5026-5027 (2008).

14. Fairclough, S. M. et al. Growth and characterization of strained and alloyed type-II ZnTe/ZnSe core-shell nanocrystals. J. Phys. Chem. C 116, 26898-26907 (2012).

15. Morello, G. et al. Intrinsic optical nonlinearity in colloidal seeded grown CdSe/ CdS nanostructures: photoinduced screening of the internal electric field. Phys. Rev. B 78, 195313 (2008).

16. Smith, A. M. \& Nie, S. Semiconductor nanocrystals: structure, properties, and band gap engineering. Acc. Chem. Res. 43, 190-200 (2010).

17. Zhong, H. \& Scholes, G. D. Shape tuning of type II CdTe-CdSe colloidal nanocrystal heterostructures through seeded growth. J. Am. Chem. Soc. 131, 9170-9171 (2009).

18. McDaniel, H., Zuo, J.-M. \& Shim, M. Anisotropic strain-induced curvature in type-II CdSe/CdTe nanorod heterostructures. J. Am. Chem. Soc. 132, 3286-3288 (2010).

19. Yang, S., Prendergast, D. \& Neaton, J. B. Strain-induced band gap modification in coherent core/shell nanostructures. Nano Lett. 10, 3156-3162 (2010).

20. McDaniel, H., Pelton, M., Oh, N. \& Shim, M. Effects of lattice strain and band offset on electron transfer rates in type-II nanorod heterostructures. J. Phys. Chem. Lett. 3, 1094-1098 (2012).

21. Halasyamani, P. S. \& Poeppelmeier, K. R. Noncentrosymmetric oxides. Chem. Mater. 10, 2753-2769 (1998).

22. Langbein, W., Hetterich, M., Grün, M., Klingshirn, C. \& Kalt, H. Picosecond luminescence dynamics in CdS/CdSe Stark superlattices. Appl. Phys. Lett. 65, 2466-2468 (1994).

23. Andreev, A. D. \& O'Reilly, E. P. Theory of the electronic structure of GaN/AlN hexagonal quantum dots. Phys. Rev. B 62, 15851-15870 (2000).

24. Madelung, O. Semiconductors: Data Handbook (Springer, 2004).

25. Simon, J. et al. Direct comparison of recombination dynamics in cubic and hexagonal GaN/AlN quantum dots. Phys. Rev. B 68, 035312 (2003).

26. Norris, D. J., Efros, A. L. \& Erwin, S. C. Doped Nanocrystals. Science 319, 1776-1779 (2008).

27. Christodoulou, S. et al. Synthesis of highly luminescent wurtzite CdSe/CdS giant-shell nanocrystals using a fast continuous injection route. J. Mater. Chem. C 2, 3439-3447 (2014).

28. Brovelli, S. et al. Nano-engineered electron-hole exchange interaction controls exciton dynamics in core-shell semiconductor nanocrystals. Nat. Commun. 2, 280 (2011).

29. Brovelli, S. et al. Dual-color electroluminescence from dot-in-bulk nanocrystals Nano Lett. 14, 486-494 (2013).

30. Kraus, R. M. et al. Room-temperature exciton storage in elongated semiconductor nanocrystals. Phys. Rev. Lett. 98, 017401 (2007).

31. Klimov, V. I. et al. Single-exciton optical gain in semiconductor nanocrystals. Nature 447, 441-446 (2007).

32. Miszta, K. et al. Hollow and concave nanoparticles via preferential oxidation of the core in colloidal core/shell nanocrystals. J. Am. Chem. Soc. 136, 9061-9069 (2014).

33. Kim, M. R. et al. Influence of chloride ions on the synthesis of colloidal branched CdSe/CdS nanocrystals by seeded growth. ACS Nano 6, 11088-11096 (2012).

34. Carbone, L. et al. Synthesis and micrometer-scale assembly of colloidal CdSe/ CdS nanorods prepared by a seeded growth approach. Nano Lett. 7, 2942-2950 (2007).

35. Sitt, A., Salant, A., Menagen, G. \& Banin, U. Highly emissive nano rod-in-rod heterostructures with strong linear polarization. Nano Lett. 11, 2054-2060 (2011).

36. Ashby, M. F. \& Brown, L. M. Diffraction contrast from spherically symmetrical coherency strains. Philos. Mag. 8, 1083-1103 (1963).

37. Williams, D. B. \& Carter, C. B. Transmission Electron Microscopy: A Textbook for Materials Science (Springer, 2009).

38. Kim, D. Y. et al. Au@Pd core-shell nanocubes with finely-controlled sizes. CrystEngComm 15, 3385-3391 (2013).

39. Galindo, P. L. et al. The peak pairs algorithm for strain mapping from HRTEM images. Ultramicroscopy 107, 1186-1193 (2007).

40. Rajadell, F., Royo, M. \& Planelles, J. Strain in free standing CdSe/CdS core-shell nanorods. J. Appl. Phys. 111, 014303 (2012).

41. De Mello Donegá, C. Formation of nanoscale spatially indirect excitons: evolution of the type-II optical character of CdTe/CdSe heteronanocrystals. Phys. Rev. B 81, 165303 (2010).

42. Jones, M., Kumar, S., Lo, S. S. \& Scholes, G. D. Exciton trapping and recombination in type II CdSe/CdTe nanorod heterostructures. J. Phys. Chem C 112, 5423-5431 (2008) 
43. Pedetti, S., Ithurria, S., Heuclin, H., Patriarche, G. \& Dubertret, B. Type-II $\mathrm{CdSe} / \mathrm{CdTe}$ core/crown semiconductor nanoplatelets. J. Am. Chem. Soc. 136, 16430-16438 (2014).

44. Pal, B. N. et al. 'Giant' CdSe/CdS core/shell nanocrystal quantum dots as efficient electroluminescent materials: strong influence of shell thickness on light-emitting diode performance. Nano Lett. 12, 331-336 (2012).

45. Nirmal, M. et al. Observation of the 'dark exciton' in CdSe quantum dots. Phys. Rev. Lett. 75, 3728-3731 (1995).

46. García-Santamaría, F. et al. Breakdown of volume scaling in auger recombination in CdSe/CdS heteronanocrystals: the role of the core - shell interface. Nano Lett. 11, 687-693 (2011).

47. Piryatinski, A., Ivanov, S. A., Tretiak, S. \& Klimov, V. I. Effect of quantum and dielectric confinement on the exciton - exciton interaction energy in type II core/shell semiconductor nanocrystals. Nano Lett. 7, 108-115 (2007).

48. Sitt, A., Della Sala, F., Menagen, G. \& Banin, U. Multiexciton engineering in seeded core/shell nanorods: transfer from type-I to quasi-type-II regimes. Nano Lett. 9, 3470-3476 (2009).

49. Steiner, D. et al. Determination of band offsets in heterostructured colloidal nanorods using scanning tunneling spectroscopy. Nano Lett. 8, 2954-2958 (2008).

50. Choi, C. L., Koski, K. J., Olson, A. C. K. \& Alivisatos, A. P. Luminescent nanocrystal stress gauge. Proc. Natl Acad. Sci. USA 107, 21306-21310 (2010).

51. Park, K., Deutsch, Z., Li, J. J., Oron, D. \& Weiss, S. Single molecule quantumconfined stark effect measurements of semiconductor nanoparticles at room temperature. ACS Nano 6, 10013-10023 (2012).

52. Htoon, H., Hollingsworth, J. A., Dickerson, R. \& Klimov, V. I. Effect of zero- to one-dimensional transformation on multiparticle auger recombination in semiconductor quantum rods. Phys. Rev. Lett. 91, 227401 (2003).

53. Park, Y.-S., Bae, W. K., Padilha, L. A., Pietryga, J. M. \& Klimov, V. I. Effect of the core/shell interface on auger recombination evaluated by single-quantumdot spectroscopy. Nano Lett. 14, 396-402 (2014).

54. Guzelturk, B. et al. Stable and low-threshold optical gain in CdSe/CdS quantum dots: an all-colloidal frequency up-converted laser. Adv. Mater. 27, 2741-2746 (2015).

55. Nasilowski, M., Spinicelli, P., Patriarche, G. \& Dubertret, B. Gradient CdSe/CdS quantum dots with room temperature biexciton unity quantum yield. Nano Lett. 15, 3953-3958 (2015).

56. Grim, J. Q. et al. Continuous-wave biexciton lasing at room temperature using solution-processed quantum wells. Nat. Nanotechnol. 9, 891-895 (2014).

57. Semonin, O. E. et al. Peak external photocurrent quantum efficiency exceeding $100 \%$ via MEG in a quantum dot solar cell. Science 334, 1530-1533 (2011).

58. Bronstein, N. D. et al. Luminescent solar concentration with semiconductor nanorods and transfer-printed micro-silicon solar cells. ACS Nano 8, 44-53 (2014).

59. Meinardi, F. et al. Large-area luminescent solar concentrators based on Stokes-shift-engineered nanocrystals in a mass-polymerized PMMA matrix. Nat. Photon. 8, 392-399 (2014).
60. Müller, M., Bounouar, S., Jöns, K. D., Glässl, M. \& Michler, P. On-demand generation of indistinguishable polarization-entangled photon pairs. Nat. Photon. 8, 224-228 (2014).

61. Ning, Z. et al. Solar cells sensitized with type-II ZnSe-CdS core/shell colloidal quantum dots. Chem. Commun. 47, 1536-1538 (2011).

\section{Acknowledgements}

F.R., J.I.C. and J.P. acknowledge financial support from MINECO project CTQ201127324 and UJI-Bancaixa P1-1B2011-01. S.B. and F.M. acknowledge support from the Cariplo Foundation (2012-0844). S.B. thanks the European Community's Seventh Framework Programme (FP7/2007-2013) under grant agreement no. 324603 for financial support (EDONHIST). The present publication is further realized with the support of the Ministero degli Affari Esteri e della Cooperazione Internazionale (IONX-NC4SOL, I.M.). K. Miszta (IIT, Italy) is acknowledged for initial discussions on the RIR samples, and W. Langbein (Cardiff University, UK) for enlightening conversations on strain and the Stark effect in CdSe/CdS superlattices.

\section{Author contributions}

The experiment designs were the result of ongoing interactions and discussions between I.M., J.I.C., G.R. and S.B. S.C. performed the synthesis, X-ray diffractometry and steady-state and nanosecond PL spectroscopy at room and low temperature. Raman spectra were measured by S.C. and analysed by S.C. and I.M. A.C. and A.G. performed the TEM measurements with further analysis in conjunction with I.M. and L.M. J.Q.G measured the ultrafast PL streak camera images at cryogenic temperatures, with G.R., T.S. and R.F.M. providing further insight into the MX emission via cw-pumped fluorescence experiments (data not shown). All MX-related results were analysed and discussed by J.Q.G., S.C., G.R., T.S., R.F.M. and I.M. G.V., S.C., F.M. and S.B. measured the fluorescence line-narrowing spectra. F.R., J.I.C. and J.P. performed the $k \cdot p$ calculations. All authors contributed to writing the manuscript.

\section{Additional information}

Supplementary Information accompanies this paper at http://www.nature.com/ naturecommunications

Competing financial interests: The authors declare no competing financial interests.

Reprints and permission information is available online at http://npg.nature.com/ reprintsandpermissions/

How to cite this article: Christodoulou, S. et al. Band structure engineering via piezoelectric fields in strained anisotropic CdSe/CdS nanocrystals. Nat. Commun. 6:7905 doi: 10.1038/ncomms8905 (2015).

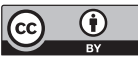

This work is licensed under a Creative Commons Attribution 4.0 International License. The images or other third party material in this article are included in the article's Creative Commons license, unless indicated otherwise in the credit line; if the material is not included under the Creative Commons license, users will need to obtain permission from the license holder to reproduce the material. To view a copy of this license, visit http://creativecommons.org/licenses/by/4.0/ 